\title{
A BINARY PROGENITOR FOR THE TYPE IIb SUPERNOVA 2011dh IN M51
}

\author{
Omar G. Benvenuto ${ }^{1,3}$, Melina C. Bersten ${ }^{2}$, and Ken'ichi Nomoto 2 \\ ${ }^{1}$ Facultad de Ciencias Astronómicas y Geofísicas, Universidad Nacional de La Plata, Paseo del Bosque S/N, \\ B1900FWA La Plata, Argentina; obenvenu@fcaglp.unlp.edu.ar \\ ${ }^{2}$ Kavli Institute for the Physics and Mathematics of the Universe, Todai Institutes for Advanced Study, \\ University of Tokyo, 5-1-5 Kashiwanoha, Kashiwa, Chiba 277-8583, Japan; melina.bersten@ipmu.jp \\ Received 2012 June 22; accepted 2012 October 27; published 2012 December 18
}

\begin{abstract}
We perform binary stellar evolutionary calculations following the simultaneous evolution of both stars in the system to study a potential progenitor system for the Type IIb supernova $2011 \mathrm{dh}$. Pre-explosion photometry as well as light-curve modeling has provided constraints on the physical properties of the progenitor system. Here, we present a close binary system (CBS) that is compatible with such constraints. The system is formed by stars of solar composition with $16 M_{\odot}+10 M_{\odot}$ on a circular orbit with an initial period of 125 days. The primary star ends its evolution as a yellow supergiant with a mass of $\approx 4 M_{\odot}$, a final hydrogen content of $\approx(3-5) \times 10^{-3} M_{\odot}$, and with an effective temperature and luminosity in agreement with the Hubble Space Telescope (HST) pre-explosion observations of SN 2011dh. These results are nearly insensitive to the adopted accretion efficiency factor $\beta$. At the time of explosion, the companion star has an effective temperature of 22,000-40,000 K, depending on the value of $\beta$, and lies near the zero-age main sequence. Considering the uncertainties in the HST pre-SN photometry, the secondary star is only marginally detectable in the bluest observed band. CBSs, as opposed to single stars, provide a natural frame to explain the properties of SN $2011 \mathrm{dh}$.
\end{abstract}

Key words: binaries: close - stars: evolution - supernovae: general - supernovae: individual (SN 2011dh)

Online-only material: color figures

\section{INTRODUCTION}

Core-collapse supernovae (CCSNe) are the explosive end of massive stars with $M_{\text {ZAMS }} \gtrsim 8 M_{\odot}$. There is a diversity in the spectroscopic and photometric properties of CCSNe which are mainly related to the ability of the progenitor to retain its outermost layers. Type II SNe, with clear $\mathrm{H}$ lines in their spectra, represent the case where a thick $\mathrm{H}$ envelope is retained before the explosion. Type $\mathrm{Ib} \mathrm{SNe}$, with no $\mathrm{H}$ lines but with clear $\mathrm{He}$ lines, have lost their $\mathrm{H}$ envelope but not the He layers. Finally, Type Ic SNe, with no $\mathrm{H}$ and He lines in the spectra, represent a more extreme case where not only the $\mathrm{H}$ but also the $\mathrm{He}$ envelopes are likely lost before the explosion. There are also transitional objects between these different types. One example is that of Type IIb SNe, which show H lines at early times but then the spectrum is transformed into that of typical $\mathrm{SNe} \mathrm{Ib}$ (see Filippenko 1997, for a classification scheme). Type IIb, $\mathrm{Ib}$, and Ic objects are collectively called striped-envelope $\mathrm{SNe}$ (Clocchiatti et al. 1996).

Progenitor models of $\mathrm{SNe}$ IIb comprising a helium star surrounded by a very thin hydrogen-rich envelope (of $\lesssim 1 M_{\odot}$ ) have been successful in explaining the observed light curves (LCs) and spectral features (Shigeyama et al. 1994; Woosley et al. 1994; Blinnikov et al. 1998). However, it is not clear which mechanism is responsible for the removal of the outer envelope before the explosion. One possibility is strong winds that occur in massive stars with $M_{\text {ZAMS }} \gtrsim 25 M_{\odot}$. Alternatively, in close binary systems (CBSs), stars are expected to exchange mass, providing an efficient mechanism to allow for the removal of outer layers. Currently, the binary channel is particularly favored for the case of SNe IIb (Eldridge et al. 2008; Claeys et al. 2011; Smith et al. 2011).

\footnotetext{
3 OGB is a member of the Carrera del Investigador Científico de la Comisión de Investigaciones Científicas de la Provincia de Buenos Aires (CIC), Argentina.
}

Additional support for the binary scenario in SNe IIb comes from the detection of a hot companion for the famous SN IIb 1993J (Maund et al. 2004). This was initially suggested by pre-explosion photometry (Aldering et al. 1994). The LC and evolutionary models of SN 1993J were also in favor of the binary channel (Nomoto et al. 1993; Podsiadlowski et al. 1993; Woosley et al. 1994). Some evidence for a companion was also reported for another SN IIb 2001ig (Ryder et al. 2006).

The Type IIb SN 2011dh was recently discovered in the nearby galaxy M51, attracting the attention of many observers because of its proximity and brightness. It was discovered almost immediately after explosion (Arcavi et al. 2011). It showed early radio and X-ray emission (Soderberg et al. 2012). Using pre-explosion images obtained from the Hubble Space Telescope (HST) archive, Maund et al. (2011) and Van Dyk et al. (2011) detected a source at the location of SN 2011dh. They derived similar values of luminosity and effective temperature for the pre-SN source. The object was consistent with a yellow supergiant (YSG) star with a radius $R \approx 270 R_{\odot}$ and without any clear evidence of a companion star contributing to the observed spectral energy distribution (SED).

At present, there is a controversy in the literature as to whether the YSG is the actual progenitor of SN 2011dh. Some authors have suggested that the exploding star should be more compact (Arcavi et al. 2011; Soderberg et al. 2012; Van Dyk et al. 2011) based on (1) a simple comparison between the early LC of SN 2011dh and SN 1993J, (2) a discrepancy between the temperature derived from an early-time spectrum and that predicted by an analytic expressions for an extended progenitor, and (3) the large shock velocity derived from radio observations.

Recently, we have performed a detailed hydrodynamical modeling of SN 2011dh using stellar evolutionary progenitors (Bersten et al. 2012). These models indicate that observations are compatible with a helium star progenitor of a mass near $4 M_{\odot}$ surrounded by a thin hydrogen-rich envelope $\left(\approx 0.1 M_{\odot}\right)$ 
with a radius of $\approx 200 R_{\odot}$ (similar to that of the detected YSG star) that underwent an explosion with an energy of $8 \times 10^{50} \mathrm{erg}$ that synthesized $0.063 M_{\odot}$ of ${ }^{56} \mathrm{Ni}$. Such large radius values are needed to reproduce the early LC of SN 2011dh without contradicting the temperatures derived from the spectra. In addition, our hydrodynamical modeling rules out progenitors with He core masses larger than $8 M_{\odot}$, which corresponds to $M_{\text {ZAMS }} \gtrsim 25 M_{\odot}$.

It is very difficult for a single star to reach these pre-SN conditions. The existence of a strong wind capable of removing most of the envelope requires a massive star of $\approx 25 M_{\odot}$ or more (Heger et al. 2003; Georgy et al. 2009), which is in contradiction with the LC models. Moreover, in order to retain a thin hydrogen-rich layer, the mass loss rate would have to be on a very narrow interval. These facts strongly suggest that the progenitor of SN 2011dh should be a component of a binary system.

However, a recent work by Georgy (2012) proposed that single YSG stars such as the one detected at the location of SN 2011dh are plausible SN progenitors. This is based on stellar evolution calculations of stars with main-sequence masses of $12-15 M_{\odot}$ under the assumption of an increased mass-loss rate several times above the standard values. However, no physical explanation is given for such an increase. Also note that a recent paper by Mauron \& Josselin (2011) found a good agreement between modern determinations of mass loss for red super giants (RSGs) and the standard mass-loss prescription (de Jager et al. 1988). Although another mass-loss formulation as proposed by van Loon et al. (2005) points toward higher mass-loss rates, this prescription seems to be applicable only to dusty stars and gives overestimates of the mass-loss rates for Galactic RSGs.

The aim of this work is to show the plausibility of the progenitor of SN 2011dh being part of a CBS with properties compatible with the pre-SN observations and the results of LC modeling. The observational properties of the remaining companion star are discussed in anticipation of future detections. Although we do not perform a complete exploration of the parameter space (stellar masses, initial orbital period, and masstransfer efficiency $\beta$ ), we show that our results are robust if we consider moderate changes of the initial conditions.

The remainder of this paper is organized as follows. In Section 2, we present a brief description of our binary stellar evolution code, paying special attention to the characteristics that enabled us to compute pre-SN models. In Section 3, we present the main results of this paper regarding the adopted binary configuration (Section 3.1), evolutionary calculations (Section 3.2), and the spectra of the components at the moment of the explosion (Section 3.3). In Section 4, we present a discussion of our results and finally, in Section 5, we provide some concluding remarks.

\section{THE STELLAR CODE}

In order to compute the evolution of the CBSs quoted above, we shall employ a code similar to that described in Benvenuto \& De Vito (2003), adapted for the case of massive stars. Briefly, this is a Henyey code that, when the star reaches Roche Lobe Over Flow (hereafter RLOF) conditions, implicitly solves not only the whole structure of the donor star but also the mass transfer rate (hereafter MTR) $\dot{M}_{1}$ in a simultaneous, fully implicit way. Such a procedure has been found to largely improve numerical stability as compared to algorithms that compute the MTR explicitly (Büning \& Ritter 2006), enabling us to reach pre-SN conditions while the donor star is losing mass at an appreciable rate with a detailed, continuous, and convergent sequence of stellar models. In detached conditions, the code works as a standard Henyey scheme.

In order to adapt the code to the case of massive stars, we have incorporated several nuclear reactions from the compilation of Caughlan \& Fowler (1988) and rewritten the difference equations to largely improve numerical stability. Also, we incorporated semiconvection following the diffusive approach presented in Langer et al. (1983) and first applied to massive stars by Langer et al. (1985). This formulation of diffusive semiconvection depends on an efficiency parameter $\alpha_{\mathrm{SC}}$ for which we assumed $\alpha_{\mathrm{SC}}=1$ as in Yoon et al. (2010). In this paper, we shall ignore the effects due to overshooting and rotation.

In semidetached conditions, we compute $\dot{M}_{1}$ following the prescription given by Ritter (1988):

$$
\dot{M}_{1}=-\dot{M}_{0} \exp \left(\frac{R_{1}-R_{L}}{H_{p}}\right),
$$

where $R_{1}$ is the radius of the donor star; $R_{L}$, the radius of a sphere with a volume equal to the corresponding Roche lobe, is computed following Eggleton (1983); $\dot{M}_{0}>0$ is a smooth function of $M_{1}$ and $M_{2}$, whereas $H_{p}=-d r / d \ln p$ (where $r$ and $p$ are the radial coordinate and pressure, respectively) is the photospheric pressure scale height. For further details, see Ritter (1988). In detached conditions, we consider stellar wind following de Jager et al. (1988). As we shall discuss in Section 3, we consider different accretion efficiencies, $\beta$, of the material transferred by the donor to the secondary, i.e., $\dot{M}_{2}=-\beta \dot{M}_{1}$, and evolve the orbit as described in Benvenuto \& De Vito (2003).

After computing the evolution of the donor star, we evolve the companion star taking into account the accretion rate it receives from the donor as described in Neo et al. (1977). We do not consider mass loss from the accreting star. As we ignore any effect of the secondary star on the donor, rather than imposing a limit on its volume, this is an usual assumption in this type of problems.

It is well known that in CBSs like those we are interested in here, the material accreted by the secondary star may have a mean molecular weight higher than that corresponding to its outer layers. This may lead to an unstable situation that induces the so-called thermohaline mixing (Kippenhahn et al. 1980). In the present version of our code, we have not included thermohaline mixing yet. In any case, it is worth remarking that Stancliffe \& Eldridge (2009) have shown that ignoring thermohaline mixing has a minor effect on the evolutionary track of secondary stars of CBSs similar to those studied here. Therefore, we expect that the main conclusions of this work will not be affected by neglecting this phenomenon.

\section{THE BINARY MODELS FOR SN 2011dh}

\subsection{The Initial Configuration}

The broadband HST pre-explosion photometry of the SN 2011dh (Maund et al. 2011; Van Dyk et al. 2011) and the LC modeling (Bersten et al. 2012) impose strong constraints on our selection of the binary parameters, and the mass of the primary, of the secondary, and the initial period. Here, we briefly discuss the motivations of our selection of these parameters before we present our results.

If the object detected in the pre-explosion image is indeed the progenitor of SN 2011 dh and it belongs to a binary system, then the primary (donor) star needs to have a luminosity $(L)$ compatible with the value derived by Maund et al. (2011) and Van 
Dyk et al. (2011) for the progenitor candidate, i.e., $\log L / L_{\odot}=$ $4.92 \pm 0.2$. This luminosity is an indication of the He core mass at the moment of the explosion which in turn is related to the mass of the star on the main sequence ( $M_{\text {ZAMS }}$ ). Maund et al. (2011) derived $M_{\text {ZAMS }}=13 \pm 3 M_{\odot}$ by comparing the luminosity with the end points of the evolutionary tracks of single stars. Note that they did not use the color information of the progenitor to derive this mass because uncertainties are expected in the color due to unknown mass-loss history. Meanwhile, Van Dyk et al. (2011) derived $M_{\text {ZAMS }}=17-19 M_{\odot}$ using both $L$ and the effective temperature $\left(T_{\text {eff }}\right)$ derived from colors and choosing the track that best matched these values, although this point does not correspond with the final position of the single star at the end of the evolution. In addition, Bersten et al. (2012) derived a helium core of $\approx 4 M_{\odot}$ from the LC modeling of SN 2011dh and firmly ruled out progenitors with $M_{\text {ZAMS }}>$ $25 M_{\odot}$. Here, we adopt an initial mass of $16 M_{\odot}$ for the donor star that is well within the ranges provided in previous studies.

After assuming a mass value for the donor star, we still have to consider the mass of the secondary star as well the orbital period of the binary. Our choice should be guided by the fact that preexplosion observations indicate that the observed portion of the spectrum is compatible with a single source. Obviously, the secondary has to be less massive than the donor star but we have to distinguish between the two cases: (1) the mass ratio is close to one and (2) the mass ratio appreciably differs from one.

Let us consider case (1): if the masses of the stars differ in (say) a few tenths of solar mass, the secondary star would be able to exhaust core hydrogen before explosion. This object would receive material coming from the donor star when it is on the Hertzsprung gap, where the shell nuclear burning around the core takes place. Calculations available in the literature as well as our own test (see Section 4) indicate that after accretion, such an object appears as an overluminous B supergiant with a $T_{\text {eff }}$ in between that of the observed YSG and that of the zero-age main sequence (ZAMS, see, e.g., Figure 5 of Claeys et al. 2011 and our Figure 13). In this case, some evidence of the secondary should have been detected in the HST pre-explosion photometry, as in the case of SN 1993J.

Case (2) results more naturally. If the secondary star has a mass appreciably lower than that of the donor, then it will still be undergoing core hydrogen burning at the moment of the explosion of the primary star. Therefore, at the moment of the explosion and in the H-R diagram (HRD), we expect the secondary to be close to the ZAMS. The object will remain hot and will emit most of the flux in the UV. As is well known, the more massive the object, the greater its luminosity on the ZAMS and therefore the greater its effect on the pre-explosion photometry. Thus, the mass value chosen for the secondary star should be low enough to have remained almost undetected. Note, however, that if the mass of the secondary is much lower than $10 M_{\odot}$, then the Kelvin-Helmholtz timescale increases appreciably and it is very likely that the system reaches common envelope conditions. Therefore, we adopt a mass of $10 M_{\odot}$ for the secondary.

Even with a mass of $10 M_{\odot}$, if the secondary object were able to undergo a conservative mass transfer and the primary star ends its evolution with a mass of $\approx 4 M_{\odot}$ as required by LC modeling, then the secondary would end its evolution with $\approx 22 M_{\odot}$ on the ZAMS. This would produce a very bright object that may not be compatible with the pre-SN observations. One possibility that we will explore in the next section is that the secondary captures a fraction $\beta$ of the material transferred, and therefore its final mass will be $\approx 10+12 \beta M_{\odot}$. Note that the accretion efficiency, $\beta$, is one of the most uncertain parameters in binary stellar evolution. Another parameter usually employed in the treatment of orbital evolution in binaries is the specific angular momentum $\alpha$ of the material lost by the system in units of the angular momentum of the primary star. We shall assume $\alpha=1$ throughout this paper.

Regarding the initial period, this has been evaluated in order for the pre-SN donor star to fall inside the error box given by the $L$ and $T_{\text {eff }}$ estimated for the progenitor candidate of SN 2011dh. Suppose that the donor star is losing mass by RLOF at the moment of the explosion, i.e., that the size of the Roche Lobe is approximately equal to the radius of the donor star. This radius can be determined using $\log L / L_{\odot}=4.92 \pm 0.2$ and $\log T_{\text {eff }}=3.78 \pm 0.02$ (Maund et al. 2011; Van Dyk et al. 2011), leading to values of $\approx 270 R_{\odot}$. If the initial masses are $16+10 M_{\odot}$ and the final masses are $4+12 M_{\odot}$, then the final orbital semiaxis is $\approx 900 R_{\odot}$ and the final period at the moment of the explosion can be estimated to be $\approx 800$ days. Finally, the initial period can be calculated using Equations (6)-(8) of Podsiadlowski et al. (2002), which relate the initial and final orbital semiaxes as a function of the initial and final masses of the system. This leads to initial periods of $\approx 120$ days. Note that if the luminosity is due to the internal structure of the star, then at pre-SN conditions a given orbital period will correspond to a given effective temperature: the larger the period, the lower the effective temperature. In the next section, we analyze in detail a system with an initial period of 125 days consistent with this first approximation. Other values are discussed in Section 5.

\subsection{Evolutionary Results}

In the previous section, we discussed our selection of the binary parameters adopted to study a possible progenitor for SN 2011dh. Here, we present our results for a CBS of solarcomposition stars with masses of $16 M_{\odot}+10 M_{\odot}$ on a circular orbit with an initial period of 125 days. As stated in Section 3.1, we analyzed different values of the mass-transfer efficiency, $\beta=$ $0.00,0.25,0.50,0.75$, and 1.00 . We computed the evolution of both stars starting with ZAMS models up to core oxygen exhaustion. The main results of these calculations are presented in Figures 1-10. Note that the evolutionary tracks corresponding specifically to the cases $\beta=0.25$ and 0.75 are only provided in the color figures available in the online journal.

As an extreme case, we show in Figure 1 the evolution of the donor and companion stars assuming $\beta=0.00$ (fully nonconservative mass transfer). This CBS undergoes class B mass transfer: the donor star fills its Roche lobe with a mass of $15.54 M_{\odot}$ well after core helium ignition (the central abundance of helium at the onset of the first RLOF is $X_{\mathrm{He}}=0.32$ ). With respect to the stellar mass, this is the main RLOF because the donor star detaches from its lobe when it has only $4.54 M_{\odot}$. Remarkably, the RLOF takes only $7.6 \times 10^{4} \mathrm{yr}$, which implies a mean MTR $\langle\dot{M}\rangle$ of $\langle\dot{M}\rangle=1.44 \times 10^{-4} M_{\odot} \mathrm{yr}^{-1}$. However the maximum mass transfer rate $\dot{M}_{\max }$ is $\dot{M}_{\max }=$ $1.90 \times 10^{-3} M_{\odot} \mathrm{yr}^{-1}$ (see below for further details on MTRs). While detaching from its Roche lobe, the donor star is still undergoing core helium burning and has increased its central abundance due to semiconvective mixing $\left(X_{\mathrm{He}}=0.39\right)$. The physical agent that sets the end of the RLOF is the outer layer's hydrogen abundance $\left.X_{\mathrm{H}}\right|_{S}$, which has fallen from its initial value of $\left.X_{\mathrm{H}}\right|_{\mathrm{S}}=0.70$ to $\left.X_{\mathrm{H}}\right|_{\mathrm{S}}=0.49$. Thus, the stellar envelope is no longer able to support its very large radius $\left(192 R_{\odot}\right)$ and starts a fast contraction, performing a blueward loop on the 


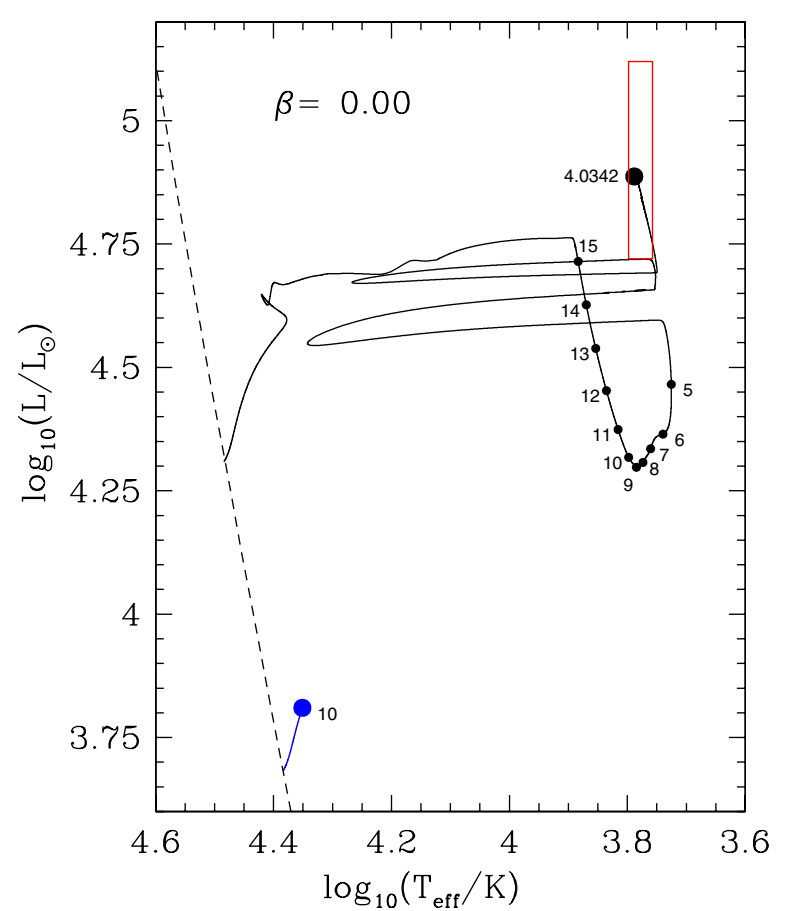

Figure 1. Evolution of the components of a close binary system of solar composition stars of $16 M_{\odot}$ and $10 M_{\odot}$ on an orbit with an initial period of 125 days assuming fully non-conservative mass transfer $(\beta=0.00)$. The solid line represents the evolutionary track of the donor star. Dots along it indicate the mass of the star during the RLOFs. The corresponding labels are in solar mass units. The star ends its evolution with a mass of $4.034 M_{\odot}$ with an effective temperature and luminosity compatible with the data observed for the object at the position of the supernova SN2011dh. While the donor star evolves from the ZAMS to pre-SN conditions, the companion star suffers a much slower evolution. Its evolutionary track is depicted with a dotted line and ends with a dot representing the conditions attained at the moment of the explosion of the primary. For comparison, the ZAMS corresponding to objects of the same composition is shown by the dashed line.

(A color version of this figure is available in the online journal.)

H-R diagram. During this loop, the core helium is exhausted. Soon, due to semiconvective mixing and nuclear shell burning, the star undergoes a second and very brief RLOF followed by another (smaller as compared to the previous) blueward loop. Finally, the star swells and again goes through an RLOF up to its final explosion. During this RLOF, the star undergoes carbon, neon, oxygen, and silicon core burning (we did not computed the silicon burning stage). During this final RLOF, the mass transferred from the donor is a relatively small amount of $0.27 M_{\odot}$ on $3.57 \times 10^{4} \mathrm{yr}\left(\langle\dot{M}\rangle=7.95 \times 10^{-6} M_{\odot} \mathrm{yr}^{-1}\right.$ and $\left.\dot{M}_{\text {max }}=2.92 \times 10^{-5} M_{\odot} \mathrm{yr}^{-1}\right)$. Note that these MTRs are far lower than that corresponding to the initial RLOF.

The evolution of the central point of the donor star in the temperature-density plane is presented in Figure 2. There, one can clearly notice the nuclear activity present in the central part of the star during each RLOF. It is remarkable that during the first RLOF, the central density and temperature remain almost constant.

In the case of $\beta=0$, the secondary star does not accrete any material and evolves as if it were an isolated object. As the main sequence lifetime of a $10 M_{\odot}$ star is far longer than that corresponding to the donor star, the companion star suffers from a very small excursion in the HRD up to the moment of explosion (see Figure 1). Let us mention here that the characteristic lifetime of isolated stars of $10 M_{\odot}$ and $16 M_{\odot}$ is $23.018 \mathrm{Myr}$ and 12.284 Myr, respectively.

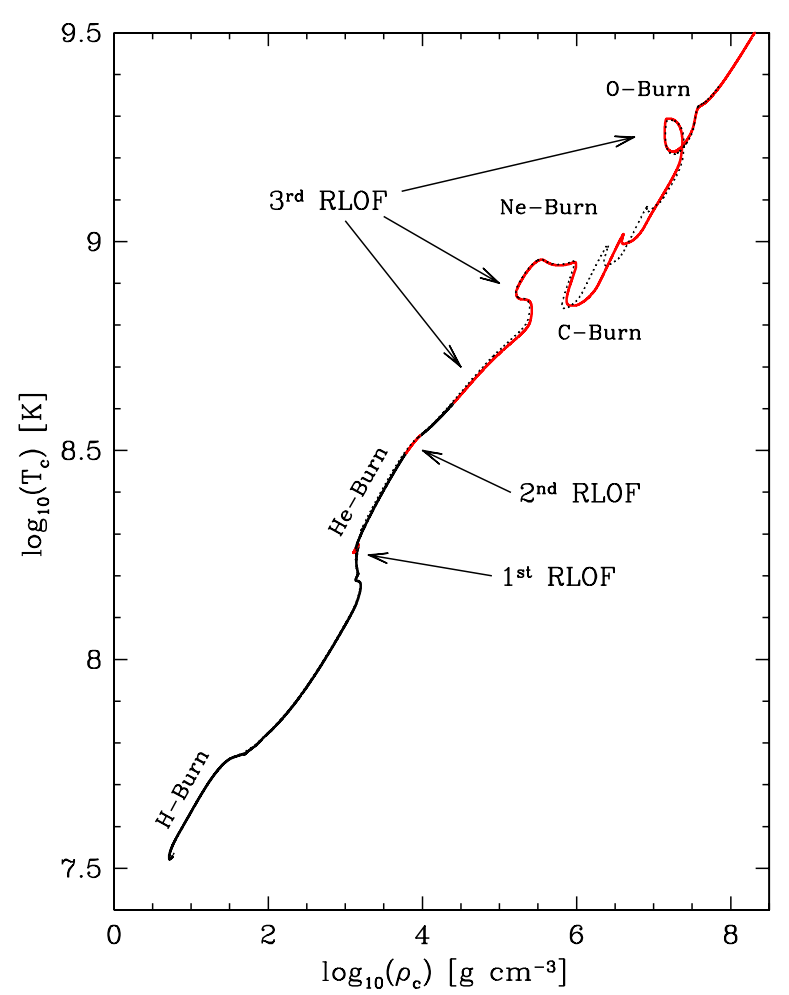

Figure 2. Evolution of the central temperature as a function of the central density. The thick line corresponds to the evolution of the donor star for the case of $\beta=0$. Its solid portion indicates detached conditions, while the dotted parts depict the evolution during RLOFs. Note that the first RLOF, in which three fourths of its initial mass are transferred, corresponds almost to a point. The thin solid line depicts the evolution of an isolated star of the same initial mass $\left(16 M_{\odot}\right)$. For other values of $\beta(>0)$, the tracks are almost indistinguishable from that corresponding to $\beta=0$ and are not included.

(A color version of this figure is available in the online journal.)

The evolution of the components of the CBS for the cases of $\beta=0.50$ and 1.00 is shown in Figures 3 and 4 , respectively, and the cases of $\beta=0.25$ and 0.75 are available in Figure 5 . An inspection of these figures is sufficient to realize that the evolution of the donor star is almost independent of the value of $\beta$ while the secondary is strongly dependent on it. In Figure 5, we show the evolutionary tracks of the donor star corresponding to the five values of $\beta$ considered in this paper. They are remarkably similar. This behavior resembles the results found in the case of low mass CBSs (De Vito \& Benvenuto 2012).

In Figure 6, we show the evolutionary tracks of the companion, accreting star as a function of $\beta$. There, for comparison, we also show the ZAMS corresponding to the initial composition of these stars. For the cases where $\beta>0$, the final position in the HRD for the accreting star is somewhat hotter and overluminous than objects of the same mass on the ZAMS. This is partially due to the fact that we have neglected thermohaline mixing in our calculations. Note that in all of the cases considered, the companion star does not fill its Roche lobe and no contact configuration is found. Thus, the CBSs studied here do not undergo any common envelope episode.

The evolution of the central point of the accreting star is shown in Figure 7. Note that the excursion of these objects in the density-temperature plane is smaller by far than the one corresponding to the donor stars. Accreting stars are not able to exhaust central hydrogen in the time spent by the donor star to reach pre-SN conditions. 


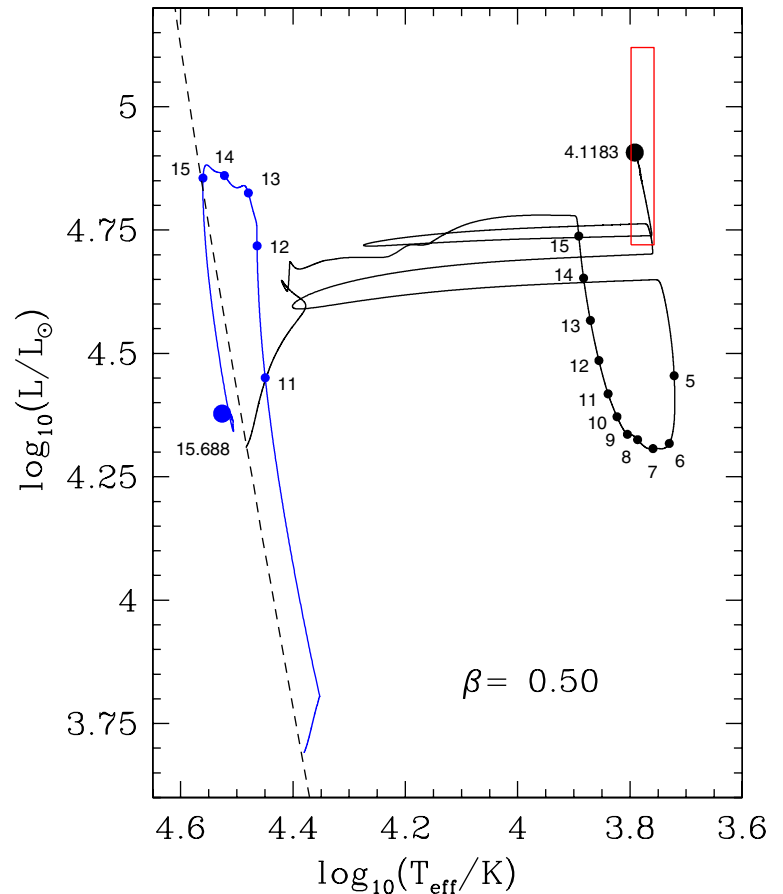

Figure 3. Same as Figure 1, but for the case of $\beta=0.50$. Lines and dots have the same meaning as there.

(A color version of this figure is available in the online journal.)

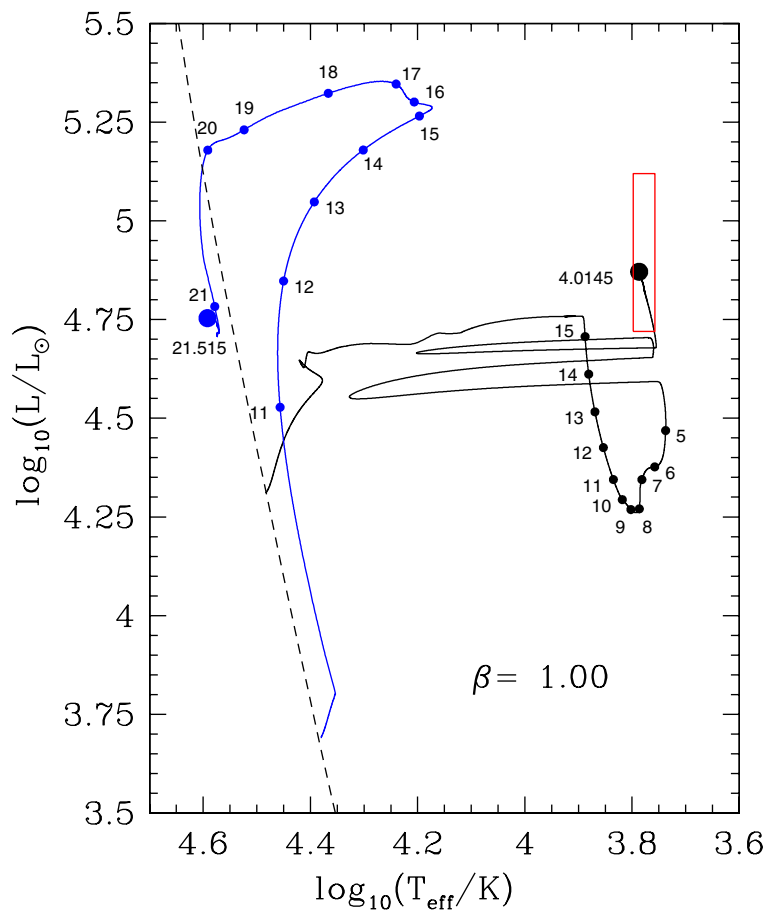

Figure 4. Same as Figure 1, but for the case of conservative mass transfer $(\beta=1.00)$. Lines and dots have the same meaning as there.

(A color version of this figure is available in the online journal.)

The evolution of the MTR from the donor star is presented in Figure 8. The only difference in the MTR evolution as a function of $\beta$ is in the time spent by the star from the end of the first RLOF to the onset of the second one for which the minimum is $\beta=0.50$. All the cases predict a third mass-transfer episode that lasts until the end of the evolution and whose MTR differs

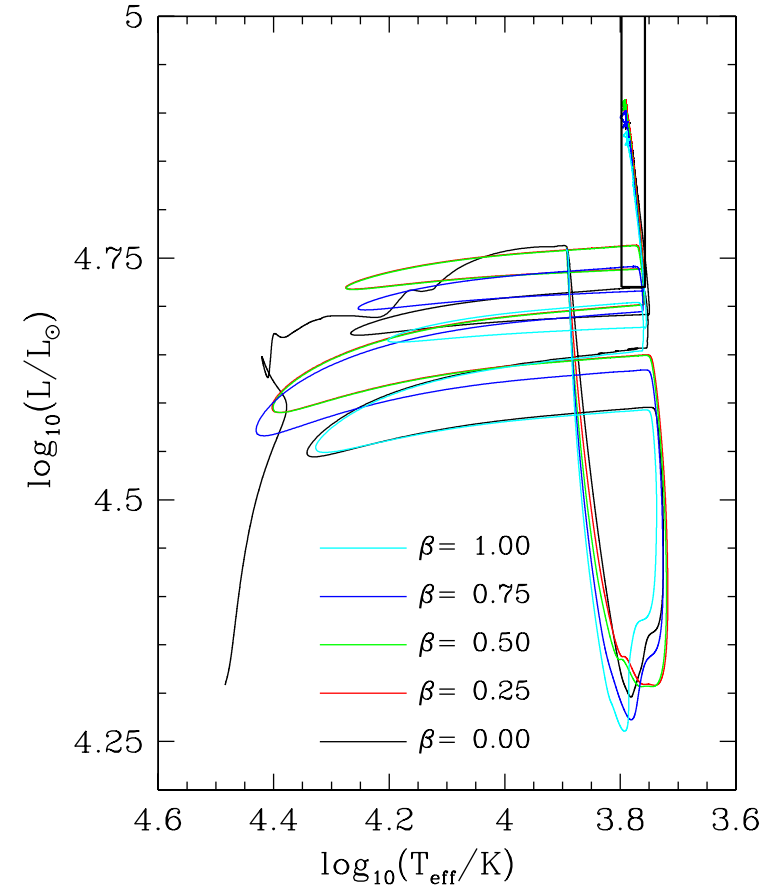

Figure 5. For ease of comparison, we show the evolutionary tracks of the donor stars for all the values of $\beta$ considered in this paper. Note that all tracks are very similar to each other.

(A color version of this figure is available in the online journal.)

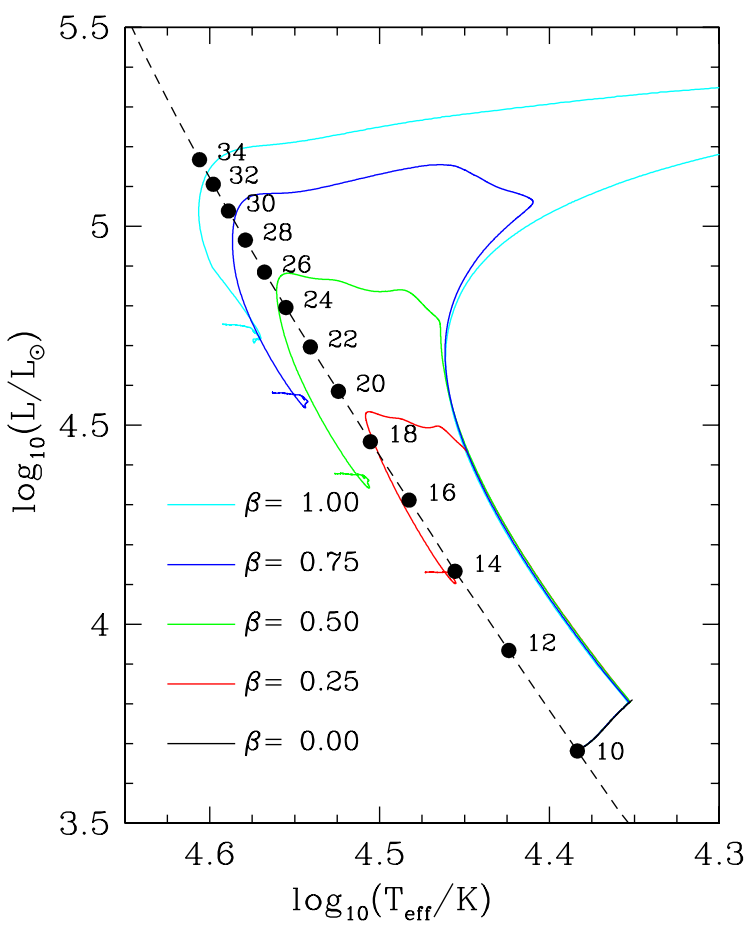

Figure 6. Same as Figure 5, but for the case of the secondary star. In sharp contrast to the case of the donor star, the evolution of the secondary star is strongly dependent on the value of $\beta$. We show the ZAMS with a dashed line on which we have indicated some values of mass (in solar units). For the cases of $\beta>0$, the final position in the HRD for the accreting star is somewhat hotter and overluminous than objects of the same mass on the ZAMS.

(A color version of this figure is available in the online journal.)

markedly from constant. Therefore, any inference on the mass of the envelope of the donor star at the time of the explosion should take into account the appropriate mass loss in this phase. 


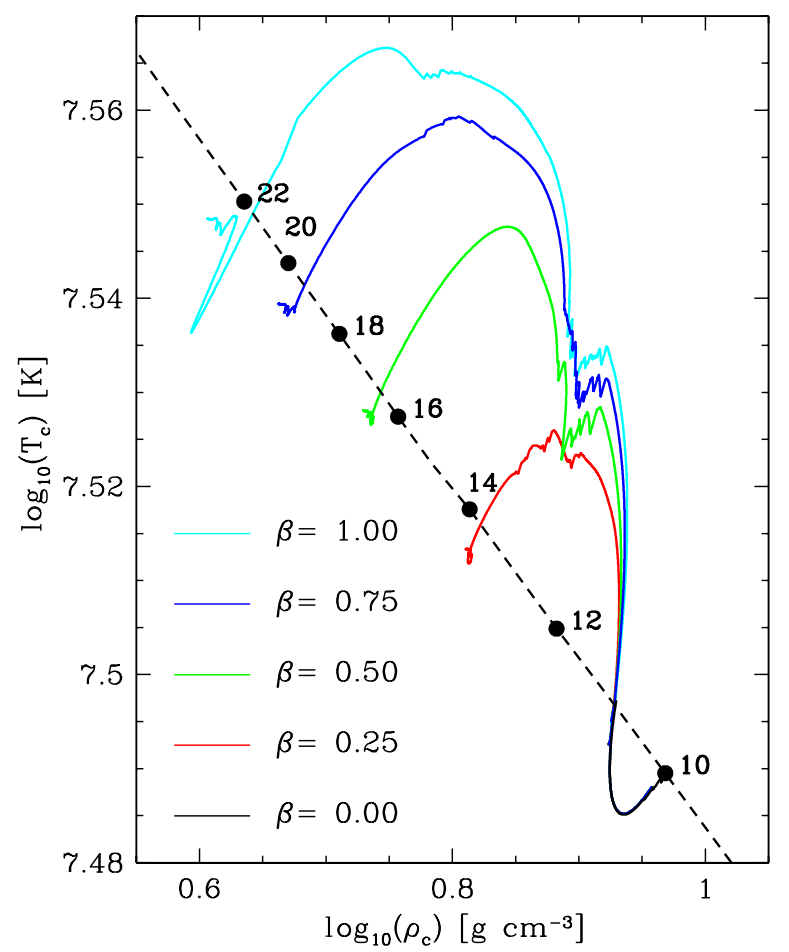

Figure 7. Evolution of the central temperature as a function of the central density for the secondary, accreting star. This is equivalent to Figure 2, which corresponds to the central evolution of the donor star, but in any case we should remark that the scale is completely different because the secondary star is still burning hydrogen during its evolution up to the explosion of the donor star.

(A color version of this figure is available in the online journal.)

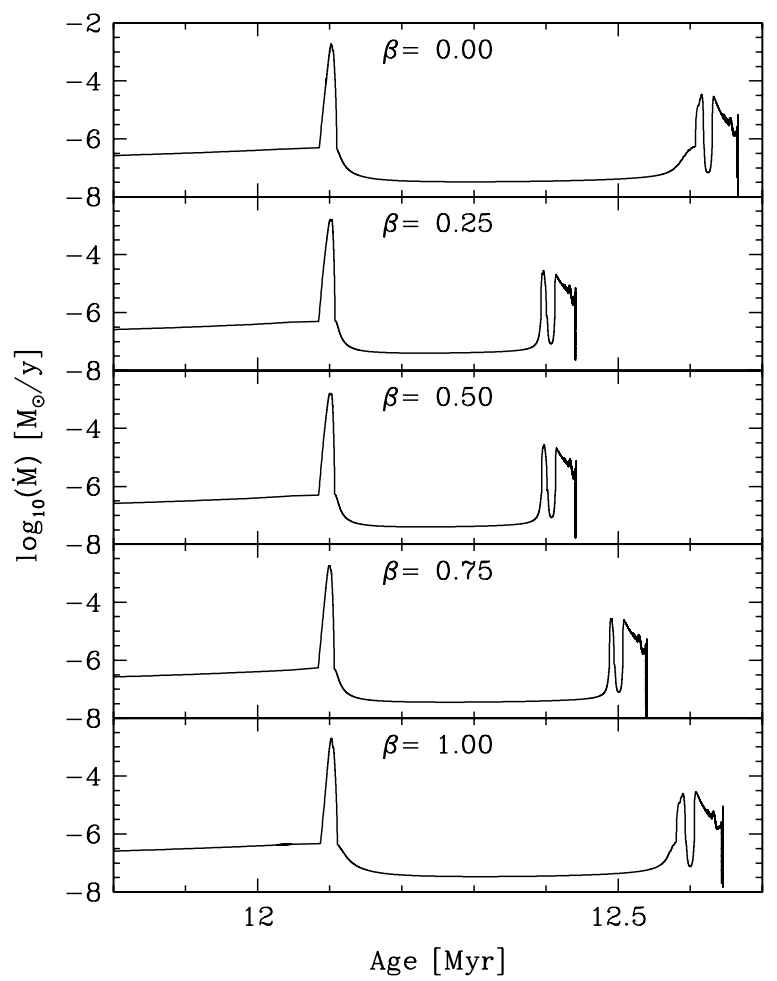

Figure 8. Mass transfer rate from the donor star as a function of time. In each panel, we show the cases for the five values of $\beta$ considered in this work. In all cases, there occur three RLOFs that have a very similar profile. The only significant change is in the time spent by the star from the end of the first RLOF to the onset of the second one. These differences are related to changes in the orbital semi-axis.

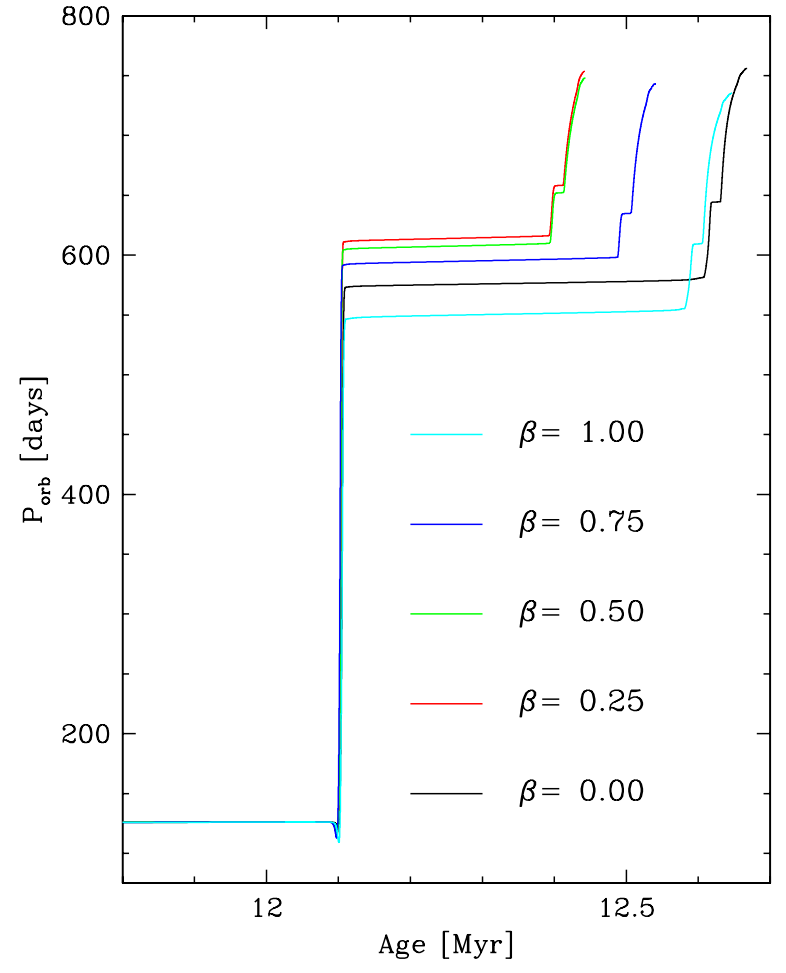

Figure 9. Temporal evolution of the orbital period for the five values of $\beta$ considered in this work.

(A color version of this figure is available in the online journal.)

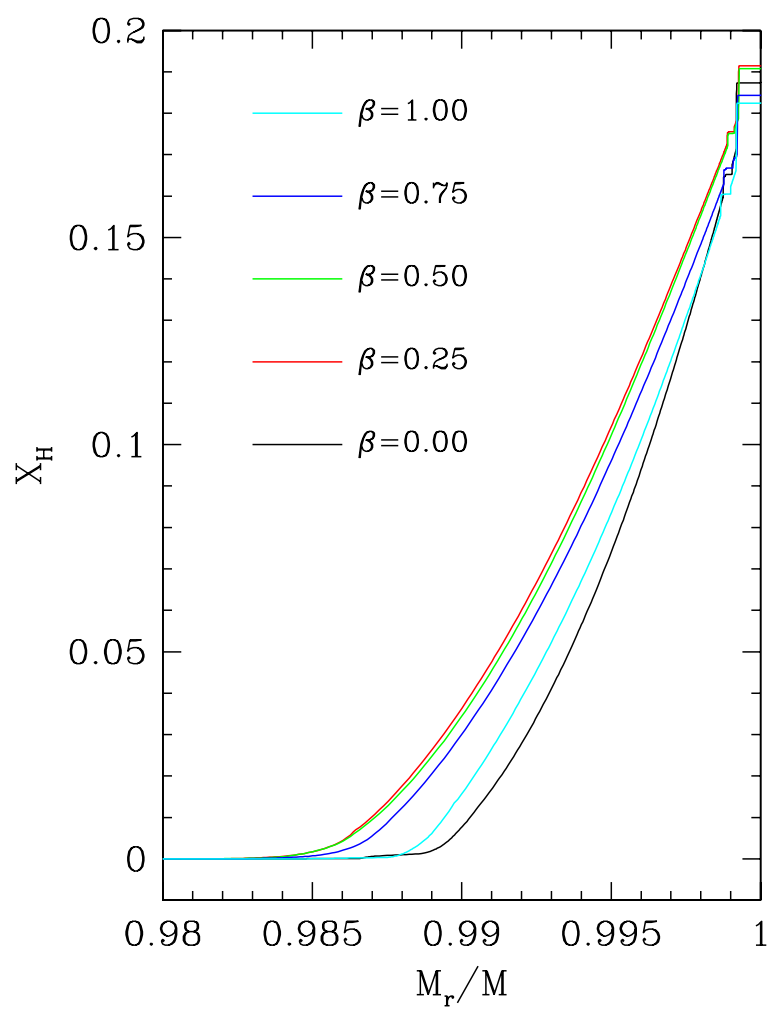

Figure 10. Final outermost hydrogen profile of pre-SN models prior to the explosion for all values of $\beta$. Note that these are very similar to each other.

(A color version of this figure is available in the online journal.) 
Table 1

Some Properties of the Orbit at the Moment of Explosion as a Function of $\beta$

\begin{tabular}{lcc}
\hline \hline$\beta$ & $\begin{array}{c}\text { Semiaxis } \\
\left(R_{\odot}\right)\end{array}$ & $\begin{array}{c}\text { Period } \\
(\text { days })\end{array}$ \\
\hline 0.00 & 841.8 & 755.84 \\
0.25 & 894.8 & 753.49 \\
0.50 & 937.6 & 747.94 \\
0.75 & 976.3 & 743.09 \\
1.00 & 1009.0 & 735.45 \\
\hline
\end{tabular}

Notes. Form left to right, we tabulate the value of $\beta$, the orbital semiaxis, and the orbital period at the moment of explosion. For all these cases, the initial period is 125 days and the orbital semiaxis is $311.5 R_{\odot}$

By analyzing the evolution of the MTR, it is possible to understand the evolution of the companion star. During the first RLOF, MTR reaches very high values $\left(\dot{M} \gtrsim 10^{-3} M_{\odot} \mathrm{yr}^{-1}\right)$. If the secondary star is able to efficiently accrete $(\beta>0.1)$ the material coming from the donor star, then it may swell appreciably, reaching low effective temperatures (see Figures 3-4). For the range of initial periods considered here $(\approx 125$ days $)$, this happens during the core helium burning of the donor star. Near the end of the first RLOF, the MTR falls down and the secondary star evolves (in the HRD) toward the ZAMS being overluminous. Thus, it has to evolve to lower luminosities, compatible with its internal nuclear energy release, keeping close to the ZAMS.

Later on, the donor star undergoes two further RLOF episodes, reaching pre-SN conditions on the last of them. While the MTR during the first RLOF is large enough to force the secondary star to swell, our calculations indicate that this is not the case in the second and third RLOFs. During these RLOFs, the MTR is about two orders of magnitude lower compared to the case of the first RLOF. So, the secondary star remains close to the ZAMS until the explosion of the donor star.

The evolution of the orbital period is not very sensitive to the value of $\beta$, as can be seen in Figure 9 (see also Table 1). This is not surprising, since Podsiadlowski et al. (2002) showed that the evolution of the orbital period of CBS depends only slightly on the accretion efficiency. Their analytic prediction for the evolution of the period as a function of the initial and final masses and the $\beta$ parameter fits almost perfectly with our numerical results.

An important result of our calculations is that despite the fact that the system is in semi-detached conditions at the moment of explosion, the donor star retains an appreciable amount of hydrogen in its outermost layers, as is shown in Figure 10. The total hydrogen content is again almost independent of the value of $\beta$ and is enough to account for the $\mathrm{H}$ lines observed in the spectra of SN 2011dh (Dessart et al. 2011). While a full exploration of the total hydrogen content of donor stars at pre-SN conditions for CBSs in general is beyond the scope of the present paper, this result strongly indicates that the total hydrogen content should be a function of the initial orbital period: the larger the period, the larger the hydrogen content. In this sense, the progenitor of SN 2011dh may be considered as a transition object.

For completeness, we present in Table 2 the main characteristics of the pre-SN object and in Table 3 those properties corresponding to the accreting, secondary star.

In summary, we have shown that a system with $16 M_{\odot}+$ $10 M_{\odot}$ and an initial period of 125 days, independent of the
Table 2

Some Properties of the Donor Star at the Moment of Explosion as a Function of $\beta$

\begin{tabular}{lcccccc}
\hline \hline$\beta$ & $\begin{array}{c}M \\
\left(M_{\odot}\right)\end{array}$ & $\begin{array}{c}\log _{10} T_{\text {eff }} \\
(\mathrm{K})\end{array}$ & $\begin{array}{c}\log _{10} L \\
\left(L_{\odot}\right)\end{array}$ & $\begin{array}{c}R \\
\left(R_{\odot}\right)\end{array}$ & $\begin{array}{c}M_{\mathrm{H}} \\
\left(10^{-3} M_{\odot}\right)\end{array}$ & $\begin{array}{c}\text { Age } \\
(\mathrm{Myr})\end{array}$ \\
\hline 0.00 & 4.034 & 3.788 & 4.886 & 245.54 & 3.869 & 12.66 \\
0.25 & 4.118 & 3.790 & 4.907 & 249.20 & 4.441 & 12.44 \\
0.50 & 4.118 & 3.791 & 4.907 & 247.73 & 4.537 & 12.44 \\
0.75 & 4.073 & 3.791 & 4.889 & 242.18 & 4.926 & 12.53 \\
1.00 & 4.014 & 3.786 & 4.871 & 242.74 & 3.465 & 12.64 \\
\hline
\end{tabular}

Notes. Form left to right, we tabulate the value of $\beta$; the mass; effective temperature; luminosity; radius; total amount of hydrogen; and the age of the pre-SN.

Table 3

Some Properties of the Secondary Star at the Moment of Explosion as a Function of $\beta$

\begin{tabular}{lccccccc}
\hline \hline$\beta$ & $\begin{array}{c}M \\
\left(M_{\odot}\right)\end{array}$ & $\begin{array}{c}\log _{10} T_{\text {eff }} \\
(\mathrm{K})\end{array}$ & $\begin{array}{c}\log _{10} L \\
\left(L_{\odot}\right)\end{array}$ & $\begin{array}{c}R \\
\left(R_{\odot}\right)\end{array}$ & $\begin{array}{c}\log _{10} \rho_{\mathrm{c}} \\
\left(\mathrm{g} \mathrm{cm}^{-3}\right)\end{array}$ & $\begin{array}{c}\log _{10} T_{\mathrm{c}} \\
(\mathrm{K})\end{array}$ & $\left.X_{\mathrm{H}}\right|_{\mathrm{c}}$ \\
\hline 0.00 & 10.000 & 4.351 & 3.810 & 5.324 & 0.929 & 7.497 & 0.426 \\
0.25 & 12.844 & 4.473 & 4.130 & 4.391 & 0.810 & 7.513 & 0.543 \\
0.50 & 15.688 & 4.526 & 4.377 & 4.570 & 0.729 & 7.522 & 0.584 \\
0.75 & 18.580 & 4.562 & 4.581 & 4.879 & 0.662 & 7.539 & 0.597 \\
1.00 & 21.515 & 4.592 & 4.753 & 5.201 & 0.606 & 7.548 & 0.613 \\
\hline
\end{tabular}

Notes. Form left to right, we tabulate the value of $\beta$; the mass; effective temperature; luminosity; radius; and the central values of density, temperature, and hydrogen abundance at the moment of explosion.

adopted value of $\beta$, predicts that the primary star ends its evolution within the region of the H-R diagram compatible with the pre-SN photometry of SN 2011dh (Maund et al. 2011; Van Dyk et al. 2011). Furthermore, at the end of the evolution, the primary star has a mass of $\approx 4 M_{\odot}$ and a hydrogen content of (3-4) $\times 10^{-3} M_{\odot}$, which is consistent with the LC modeling (Bersten et al. 2012) and the SN IIb classification of SN 2011dh.

\subsection{Pre-supernova Spectral Energy Distribution}

Our model calculations predict that the primary star ends its evolution with properties ( $L$ and $T_{\text {eff }}$ ) compatible with those inferred for the pre-explosion object located at the SN position (Maund et al. 2011; Van Dyk et al. 2011). At the same time, the secondary star has a luminosity that, depending on the value of $\beta$, could be comparable with that of the primary star and therefore produce a detectable effect on the pre-SN SED. In all of the cases, the secondary star is found to be significantly hotter than the donor star. Indeed, for high enough effective temperatures, the effect of the secondary would only be appreciable in the bluest available photometric band.

We thus study here the effect of the secondary on the SED of the system for different values of $\beta$, and compare this with the HST pre-explosion photometry. To calculate the SED for each star of the binary system as well as the composed SED, we used atmospheric models for solar composition provided by Kurucz (1993). The model spectrum of each star was obtained by linearly interpolating at the values of $T_{\text {eff }}$ and surface gravity $(g)$ given in Tables 2 and 3. The observed fluxes were computed by multiplying the models by $(R / d)^{2}$, where $d$ is the distance to M51 assumed to be 7.1 Mpc (Takáts \& Vinkó 2006). The sum of the SEDs was used to compute synthetic photometry through the $H S T$ transmission filters. ${ }^{4}$

\footnotetext{
4 http://www.stsci.edu/hst/wfc3/ins_performance/filters/
} 

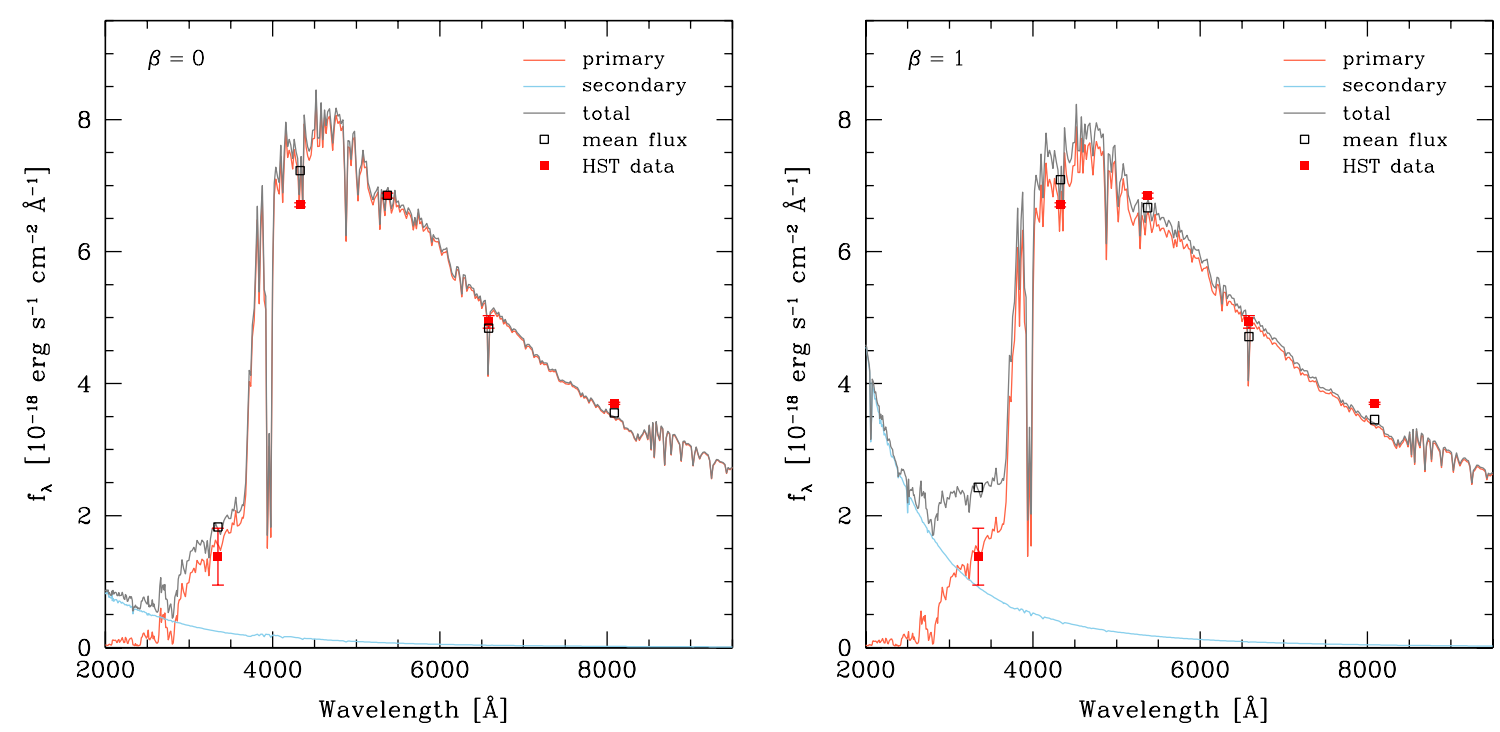

Figure 11. Spectra of the donor and accreting stars (shown dotted and dashed lines respectively). The solid line represents the addition of both spectra which, in turn, should represent the observed one. We show the results corresponding to fully non-conservative and conservative cases ( $\beta=0$ and 1 , respectively). The mean synthetic (hollow squares) and observed fluxes (solid squares) in each bandpass are included in the figure. The secondary star is significantly bluer than the primary at the time of explosion. Its contribution to the total flux is non-negligible only for the bluest observed band, $F 336 W$. See Section 3.3 for further discussion.

(A color version of this figure is available in the online journal.)

Figure 11 shows the resulting SED of each star and their sum for $\beta=0$ and 1 . Mean synthetic and observed fluxes are compared for all bands. Observed fluxes were obtained from the tabulated magnitudes given by Van Dyk et al. (2011) and zero points in the Vega system calculated using the Alpha Lyrae SED provided by Bohlin (2007). Note that the contribution of the secondary star is significant only for the bluest observed band, F336W. The increase in the F336W flux due to the presence of the secondary is $16 \%, 22 \%, 32 \%, 45 \%$, and $62 \%$ for the cases $\beta=0.00,0.25,0.50,0.75$, and 1.00 , respectively.

For the rest of the observed $H S T$ bands, the contribution of the secondary is $<6 \%$, i.e., for wavelengths $\lambda \geqslant 4000 \AA$, the spectra are completely dominated by the light coming from the donor, pre-SN star. In this wavelength range, the available observations on four photometric bands place no constraint on the properties of the accreting star. On the contrary, the bluest filter, with a maximum transmittance at $\lambda \approx 3400 \AA$, partially detects the red tail of the spectrum of the accreting star.

For the filters with transmittance at $\lambda \geqslant 4000 \AA$, the agreement between calculations and observations is very good, especially considering that we have not adjusted any parameter. For the case of the bluest filter, F336W, the contribution of the secondary to the total flux is not negligible. If we consider the measurement uncertainty in this band, then we find that the secondary is detectable only at the $2 \sigma$ level in the most extreme case $(\beta=1)$. The contribution is further decreased to the $0.6 \sigma$ level when completely non-conservative mass accretion $(\beta=0)$ is considered.

Remarkably, irrespective of the value of $\beta$, at the moment of the explosion, the secondary star is so hot that its light has barely been detected. Thus, quite unfortunately, the characteristics of the secondary star are very poorly constrained by the presently available data.

\section{DISCUSSION}

In the previous section, we showed that a properly chosen binary configuration can explain very well the proposed YSG progenitor of SN 2011dh. Our models also predict that the total and hydrogen masses of the donor star at the moment of the

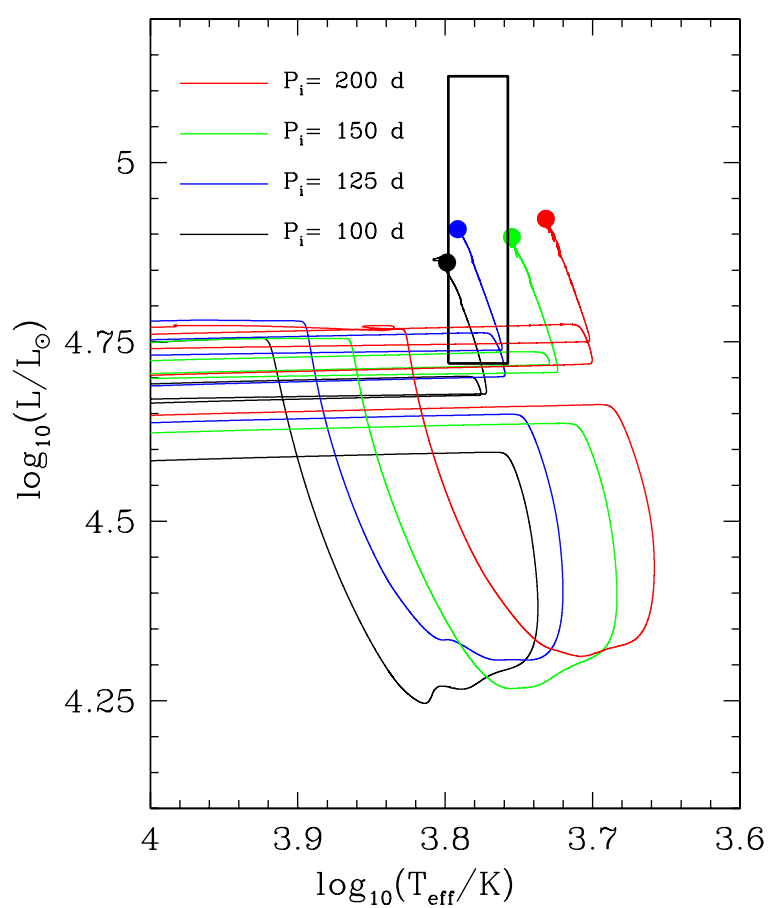

Figure 12. Red part of the evolutionary tracks for solar composition stars of binary systems with masses of $16 M_{\odot}+10 M_{\odot}$, a fixed value of $\beta=0.50$ and different values of the initial orbital period. The larger the initial period, the lower the effective temperature attained by the pre-SN. For $P_{i}=125$ days, we find the pre-SN object inside the error box allowed by photometry prior to the explosion without the need for any fine tuning.

(A color version of this figure is available in the online journal.)

explosion are consistent with the results of LC modeling and the SN IIb classification.

A complete exploration of the parameter space of initial stellar masses and orbital periods is not the intended scope of this paper. Nevertheless, we briefly test whether our results are robust if we consider moderate changes of the initial parameters. For example, Figure 12 shows the sensitivity of the evolutionary track of the primary star on small variations of the initial 


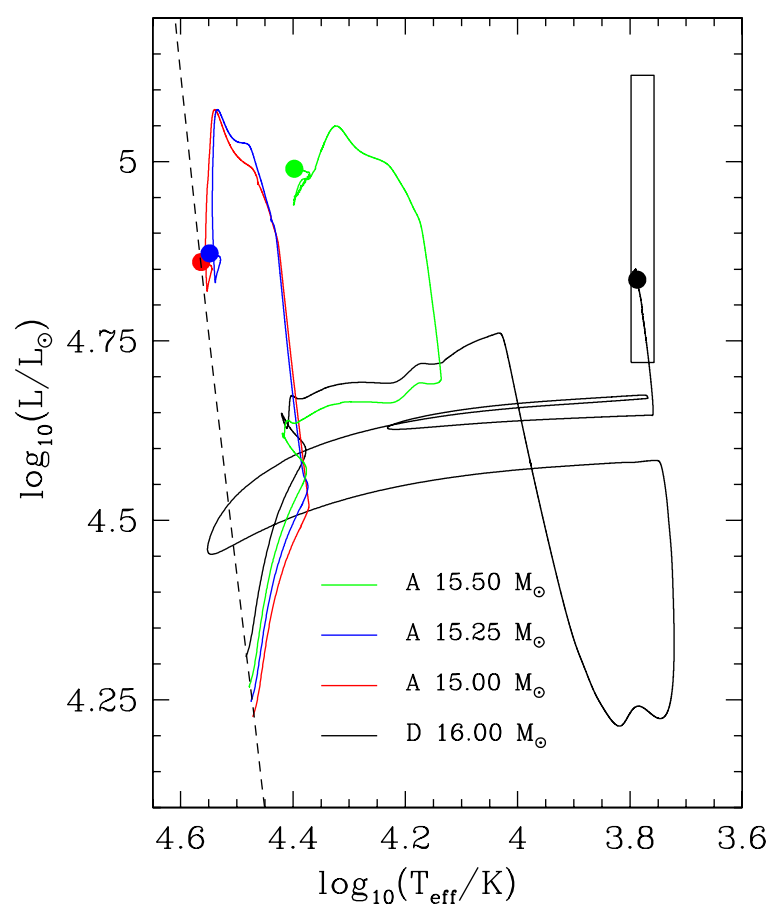

Figure 13. Evolution of binary systems with a mass of the primary of $16 M_{\odot}$, $\beta=0.50$ and different values for the initial mass of the companion. Labels $\mathrm{A}$ and D stand for accretor and donor stars, respectively. For simplicity, we computed the evolution of the donor with the $15 M_{\odot}$ companion and assumed it to be the same for the other companion masses. For this donor initial mass value, secondary stars with masses up to $\approx 15.25 M_{\odot}$ fall close to the ZAMS at the moment of the explosion of the donor star and most of its light will be emitted on the blue part of the spectrum. However, if the secondary star has an initial mass of $15.50 \mathrm{M}_{\odot}$, at the supernova event it will have a much lower effective temperature. This is not compatible with the available observations of the progenitor of SN 2011dh.

(A color version of this figure is available in the online journal.)

period, $P_{i}$ between 100 and 200 days, for the same configuration presented in the previous section, i.e., $16+10 M_{\odot}$, and $\beta=0.5$. Within such a range of initial periods, the donor star ends its evolution as a YSG close to the region of the HRD allowed by the pre-SN photometry. The evolutionary track of the secondary is not shown in Figure 12 because it is not sensitive to the initial period in the range under study.

The effect of changes in the mass of the secondary star on the binary evolution is shown in Figure 13. Three values were considered, $M_{2}=15,15.25$, and $15.50 M_{\odot}$. In all of the cases, we assumed a value of $\beta=0.5$ and a mass of the primary star of $16 M_{\odot}$, as in our previous models. The initial period was modified to make the end point of the donor star match the region allowed by the pre-SN photometry. Following the criteria described in Section 3.1, the adopted initial period for this test was of 50 days. From the figure, it is clear that only for the case of $M_{2}=15.5 M_{\odot}$, where the mass ratio is closest to one, does the secondary star move away from the ZAMS appreciably at the moment of the explosion of the primary. This is because the secondary has exhausted its hydrogen core before the beginning of the first RLOF. Consequently, the secondary ends with a color that is redder than that of lower-mass stars and is expected to contribute significantly to the observed pre-SN photometry. Indeed, the flux of the secondary in the bluest observed band, F336W, is 2.5 times larger than that of the primary star, which would lead to a $8.5 \sigma$ detection. For the rest of the HST bands, the contribution of the secondary is within $6 \%-30 \%$. While not compatible with the case of SN 2011dh, this configuration may be applicable to SN 1993J, whose pre-SN observations showed evidence of a companion star (Stancliffe \& Eldridge 2009).

Secondary stars of slightly lower initial masses (15 and $15.25 M_{\odot}$ ) remain near the ZAMS and end as hot stars of similar luminosity to the primary. Stars of masses between 10 and 15 will also remain as blue objects near the ZAMS. Because of the high effective temperature, most of the flux from the secondary is emitted in the UV, away from the bandpasses of the preexplosion imaging. However, if the luminosity is high enough, then the secondary may produce a detectable effect in the optical range. For instance, with masses of 15 and $15.25 M_{\odot}$, the flux of the secondary in the $\mathrm{F} 336 \mathrm{~W}$ band would be comparable to that of the primary star. The level of detection of the secondary in these cases would be close to $3.5 \sigma$. The final luminosity of the secondary also depends on the assumed value of $\beta$. Lower initial masses could result in similarly high luminosities with values of $\beta$ close to unity. Unfortunately, it is not possible to break such degeneracy based solely on the available pre-explosion photometry. Future observations of the possible secondary star after the SN fades from sight can shed light on this matter.

The initial mass of $16 M_{\odot}$ for the primary star leads to a progenitor with the right characteristics as derived from preexplosion photometry, LC modeling, and spectral classification. Therefore, our choice of initial masses and orbital period is by no means unique but it allows us to prove that a CBS is a plausible progenitor for SN 2011 dh and other SNe IIb. Moreover, the right progenitor properties are achieved in a self-consistent manner, independent of the detailed initial conditions.

In the past few years, some progenitors of SNe II have been associated with YSG stars, e.g., Type IIP SN 2008cn (EliasRosa et al. 2009), Type IIL SN 2009kr (Fraser et al. 2010; EliasRosa et al. 2010), and the SN studied here. The explosion of a YSG is not compatible with the theoretical prediction of single stellar evolution. Motivated by this apparent discrepancy and the lack of evidence of a companion for SN 2011dh, Georgy (2012) studied the effect of an increased mass-loss rate on the final properties of stars with initial masses of $12-15 M_{\odot}$. Assuming rates several times higher than the standard values, they found that it was possible to explain the explosion of single stars of relatively low mass in the yellow area of the HRD. In particular, the proposed scenario could explain the progenitor of SN 2011dh. However, no physical explanation was given for such an increased mass-loss process.

Note that the evolutionary tracks of isolated stars are strongly dependent upon the details of mass loss. In this sense, explaining the position of the progenitor of SN 2011dh in the HRD would require some degree of fine tuning. The binary scenario instead provides a self-consistent picture that naturally explains why the star remains as YSG for long periods of time until the explosion. In the case of CBS evolution, we find that irrespective of the mass transfer efficiency, the donor star undergoes a final RLOF before igniting carbon and so it is still transferring mass to the companion at the moment of explosion. During the final RLOF, the donor star transfers a small amount of material. So, the orbit and its Roche lobe enlarge very little while the donor star tends to swell as a consequence of nuclear shell burning. This precludes the donor star to attain lower effective temperatures and makes it evolve to higher luminosities in a way that is equivalent to the evolution of giant stars with extended outer convective zones. In any case, the observed effective temperature of the proposed progenitor of SN 2011dh strongly indicates the initial orbital period, but no other parameter has to be adjusted for the pre-SN 
object to fall inside the observational error box. The YSG nature of the progenitor is thus a direct consequence of close binary evolution.

Recently, Chevalier \& Soderberg (2010) suggested a division of SNe IIb into compact (cIlb; $R \sim 10^{11} \mathrm{~cm}$ ) and extended (eIIb; $R \sim 10^{13} \mathrm{~cm}$ ) subtypes essentially based on radio LC properties. Compact objects were proposed to have smaller hydrogen masses, roughly below $0.1 M_{\odot}$. Claeys et al. (2011) used this criterion and their own CBS evolutionary code to analyze the range of periods (for $P_{i}>1000$ days) and initial masses needed to produce extended SNe IIb. However, our calculations show that it is possible to have an extended progenitor $\left(R \approx 250 R_{\odot}\right)$ with an $\mathrm{H}$ mass of $<0.1 M_{\odot}$. Therefore, if the cIIb and eIIb subtypes correspond to physically distinct progenitors, then the division criterion may need to be revised. In addition, Yoon et al. (2010) also analyzed the space of parameters in CBSs to produce Type Ib/Ic SNe using very different initial periods of $\lesssim 8$ days, as compared with the ones adopted here. They found that some of their models predict a thin hydrogen layer of $\lesssim 0.01 M_{\odot}$ with a compact structure. This channel of production of SNe IIb is different from the one we have presented and leads to explosions far away from the YSG regime.

\section{CONCLUSIONS}

With the aim of providing a description of the progenitor of SN 2011dh, we have studied the evolution of CBSs of solar composition stars with masses of $16 M_{\odot}+10 M_{\odot}$. We considered an initial period of 125 days and different efficiencies $(\beta)$ of the mass transfer process. We followed the simultaneous evolution of the donor and accreting stars from the ZAMS up to the oxygen core exhaustion of the donor. We found that the donor star, independent of $\beta$, ends its evolution with effective temperature and luminosity consistent with the YSG object detected in the HST pre-SN photometry. The exploding star has a mass $M \approx 4 M_{\odot}$, a radius $R \approx 250 R_{\odot}$, and an outermost layer containing $(3-5) \times 10^{-3} M_{\odot}$ of hydrogen. This is generally consistent with the type IIb classification and the results of LC modeling of SN 2011dh by Bersten et al. (2012). These results are a natural consequence of the close binary evolution and require no external adjustment of any physical condition.

Regarding the accretion efficiency, $\beta$, we found that (1) the evolution of the donor star is almost independent of $\beta$ while the secondary strongly depends on it, and (2) the evolution of the orbital period, the MTR, and the total hydrogen content are almost independent of the value of $\beta$.

Our calculations indicate that the donor star is losing mass at the moment of the explosion with rates that differ markedly from constant. Inferences on the mass of the donor star at the time of the explosion should take into account the appropriate mass loss in this phase. We also found some indication that the total hydrogen content may be a function of the initial orbital period, with larger period producing a larger the hydrogen content. A more detailed study of this point is left for future work.

Note that the structure of the donor star at the moment of the explosion is consistent with an extended SN IIb but with very little $\mathrm{H}$ mass $\left(<0.1 M_{\odot}\right)$.

We analyzed the effect of the secondary star on the observed $H S T$ pre-explosion photometry. For all of the values of $\beta$, at the moment of the explosion of the donor, the secondary star is still near the ZAMS. This is a direct consequence of our assumption that the object has a mass appreciably lower than that of the donor. The effective temperature of the companion is far higher than that of the donor, with values within $22,000-40,000 \mathrm{~K}$.
Thus, the largest contribution to the flux of the system from the secondary is in the bluest observed band, F336W, producing a marginal detection of $0.6 \sigma-2 \sigma$ level depending on the value of $\beta$. Unfortunately, the available HST pre-SN observations are not very suitable to constrain the properties of the secondary.

The ultimate proof of the binary nature of SN 2011dh must come from the possible detection of a very hot star once the SN light fades enough. This situation would be similar to what occurred with SN 1993J but with different properties of the companion. In any case, we should remark that detecting the companion star of SN 2011dh would provide valuable information on the efficiency of the mass transfer process and evolution of massive CBSs in general.

O.G.B. wants to thank Professor Juan Carlos Forte for his help in getting funds that enabled him to visit the IPMU. The funds were provided by a PIP 712 of the Comisión de Investigaciones Científicas y Técnicas (CONICET), Argentina. This research has been supported in part by the Grant-in-Aid for Scientific Research of MEXT (22012003 and 23105705) and JSPS (23540262) and by World Premier International Research Center Initiative, MEXT, Japan.

\section{REFERENCES}

Aldering, G., Humphreys, R. M., \& Richmond, M. 1994, AJ, 107, 662

Arcavi, I., Gal-Yam, A., Yaron, O., et al. 2011, ApJL, 742, 18

Benvenuto, O. G., \& De Vito, M. A. 2003, MNRAS, 342, 50

Bersten, M. C., Benvenuto, O. G., Nomoto, K., et al. 2012, ApJ, 757, 31

Blinnikov, S. I., Eastman, R., Bartunov, O. S., Popolitov, V. A., \& Woosley, S. E. 1998, ApJ, 496, 454

Bohlin, R. C. 2007, in ASP Conf. Ser. 364, The Future of Photometric, Spectrophotometric and Polarimetric Standardization, ed. C. Sterken (San Francisco, CA: ASP), 315

Büning, A., \& Ritter, H. 2006, A\&A, 445, 647

Caughlan, G. R., \& Fowler, W. A. 1988, ADNDT, 40, 283

Chevalier, R. A., \& Soderberg, A. M. 2010, ApJL, 711, 40

Claeys, J. S. W., de Mink, S. E., Pols, O. R., Eldridge, J. J., \& Baes, M. 2011, A\&A, 528, A131

Clocchiatti, A., Wheeler, J. C., Brotherton, M. S., et al. 1996, ApJ, 462, 462

de Jager, C., Nieuwenhuijzen, H., \& van der Hucht, K. A. 1988, A\&AS, 72, 259

Dessart, L., Hillier, D. J., Livne, E., et al. 2011, MNRAS, 414, 2985

De Vito, M. A., \& Benvenuto, O. G. 2012, MNRAS, 421, 2206

Eggleton, P. P. 1983, ApJ, 268, 368

Eldridge, J. J., Izzard, R. G., \& Tout, C. A. 2008, MNRAS, 384, 1109

Elias-Rosa, N., Van Dyk, S. D., Li, W., et al. 2009, ApJ, 706, 1174

Elias-Rosa, N., Van Dyk, S. D., Li, W., et al. 2010, ApJL, 714, 254

Filippenko, A. V. 1997, ARA\&A, 35, 309

Fraser, M., Takáts, K., Pastorello, A., et al. 2010, ApJL, 714, 280

Georgy, C. 2012, A\&A, 538, L8

Georgy, C., Meynet, G., Walder, R., Folini, D., \& Maeder, A. 2009, A\&A, 502,611

Heger, A., Fryer, C. L., Woosley, S. E., Langer, N., \& Hartmann, D. H. 2003, ApJ, 591, 288

Kippenhahn, R., Ruschenplatt, G., \& Thomas, H.-C. 1980, A\&A, 91, 175

Kurucz, R. L. 1993, Kurucz CD-ROM, c1993 December 4 (Cambridge, MA: Smithsonian Astrophysical Observatory)

Langer, N., El Eid, M. F., \& Fricke, K. J. 1985, A\&A, 145, 179

Langer, N., Fricke, K. J., \& Sugimoto, D. 1983, A\&A, 126, 207

Maund, J. R., Fraser, M., Ergon, M., et al. 2011, ApJL, 739, 37

Maund, J. R., Smartt, S. J., Kudritzki, R. P., Podsiadlowski, P., \& Gilmore, G. F. 2004, Natur, 427, 129

Mauron, N., \& Josselin, E. 2011, A\&A, 526, A156

Neo, S., Miyaji, S., Nomoto, K., \& Sugimoto, D. 1977, PASJ, 29, 249

Nomoto, K., Suzuki, T., Shigeyama, T., et al. 1993, Natur, 364, 507

Podsiadlowski, P., Hsu, J. J. L., Joss, P. C., \& Ross, R. R. 1993, Natur, 364, 509

Podsiadlowski, P., Rappaport, S., \& Pfahl, E. D. 2002, ApJ, 565, 1107

Ritter, H. 1988, A\&A, 202, 93

Ryder, S. D., Murrowood, C. E., \& Stathakis, R. A. 2006, MNRAS, 369, L32 
Shigeyama, T., Suzuki, T., Kumagai, S., et al. 1994, ApJ, 420, 341

Smith, N., Li, W., Filippenko, A. V., \& Chornock, R. 2011, MNRAS, 412, 1522

Soderberg, A. M., Margutti, R., Zauderer, B. A., et al. 2012, ApJ, 752, 78

Stancliffe, R. J., \& Eldridge, J. J. 2009, MNRAS, 396, 1699

Takáts, K., \& Vinkó, J. 2006, MNRAS, 372, 1735 van Loon, J. T., Cioni, M.-R. L., Zijlstra, A. A., \& Loup, C. 2005, A\&A, 438,273

Van Dyk, S. D., Li, W., Cenko, S. B., et al. 2011, ApJL, 741, 28

Woosley, S. E., Eastman, R. G., Weaver, T. A., \& Pinto, P. A. 1994, ApJ, 429, 300

Yoon, S.-C., Woosley, S. E., \& Langer, N. 2010, ApJ, 725, 940 